\title{
Mass-radius relationships for exoplanets
}

D.C. Swift, J.H. Eggert, D.G. Hicks, S. Hamel, K. Caspersen, E. Schwegler, G.W. Collins Lawrence Livermore National Laboratory, 7000 East Avenue, Livermore, California 94550, U.S.A.

N. Nettelmann

Institut für Physik, Universität Rostock, D-18051 Rostock, Germany

G.J. Ackland

Centre for Science at Extreme Conditions, School of Physics, University of Edinburgh, Edinburgh, EH9 3JZ, Scotland, U.K.

January 26, 2010, modified April 13, 2011 and September 12, 2011 - LLNL-JRNL421766

Received __; accepted _ 


\begin{abstract}
For planets other than Earth, particularly exoplanets, interpretation of the composition and structure depends largely on comparing the mass and radius with the composition expected given their distance from the parent star. The composition implies a mass-radius relation which relies heavily on equations of state calculated from electronic structure theory and measured experimentally on Earth. We lay out a method for deriving and testing equations of state, and deduce mass-radius and mass-pressure relations for key, relevant materials whose equation of state is reasonably well established, and for differentiated Fe/rock. We find that variations in the equation of state, such as may arise when extrapolating from low pressure data, can have significant effects on predicted massradius relations, and on planetary pressure profiles. The relations are compared with the observed masses and radii of planets and exoplanets, broadly supporting recent inferences about exoplanet structures. Kepler-10b is apparently 'Earthlike,' likely with a proportionately larger core than Earth's, nominally $2 / 3$ of the mass of the planet. CoRoT-7b is consistent with a rocky mantle over an Fe-based core which is likely to be proportionately smaller than Earth's. GJ 1214b lies between the mass-radius curves for $\mathrm{H}_{2} \mathrm{O}$ and $\mathrm{CH}_{4}$, suggesting an 'icy' composition with a relatively large core or a relatively large proportion of $\mathrm{H}_{2} \mathrm{O}$. CoRoT-2b is less dense than the hydrogen relation, which could be explained by an anomalously high degree of heating or by higher than assumed atmospheric opacity. HAT-P-2b is slightly denser than the mass-radius relation for hydrogen, suggesting the presence of a significant amount of matter of higher atomic number. CoRoT-3b lies close to the hydrogen relation. The pressure at the center of Kepler-10b is $1.5_{-1.0}^{+1.2} \mathrm{TPa}$. The central pressure in CoRoT-7b is probably close to $0.8 \mathrm{TPa}$, though may be up to $2 \mathrm{TPa}$. These pressures are accessible by pla-
\end{abstract}


nar shock and ramp loading experiments at large laser facilities. The center of HAT-P-2b is probably around $210 \mathrm{TPa}$, in the range of planned National Ignition Facility experiments, and that of CoRoT-3b around $1900 \mathrm{TPa}$.

Subject headings: Equation of state - Planets and satellites: composition - Planets and satellites: interior 


\section{Introduction}

Planets outside the solar system have been detected since 1992 (Wolszan \& Frail 1992) from the Doppler shift of spectral features in emission from the star, which determines the orbital period and places a constraint on the mass of the planet (Mayor \& Queloz 1995). Since 1999, the presence of exoplanets has also been deduced from their transit across the face of the parent star (Henry et al 2000). The fraction of light blocked by the planet allows the radius of the planet to be deduced as a function of the radius of the star (Charbonneau et al 2000; Carter et al 2011). Subsequently, several hundred exoplanets have been detected at different distances from their stars, and the precision with which mass and radius have been deduced has increased for some exoplanets to better than $10 \%$ in both mass and radius (Schneider 2011).

In 2001, light from an exoplanet was detected directly (Charbonneau et al 2002), opening the window to studies of exoplanet structure through the composition and temperature of the surface or atmosphere. However, inferences about the composition and structure rely on the comparison of mass and radius with planets within the solar system. With the exception of Earth, which is currently the only planet for which seismic data exist, interpretations of the internal structure of the planets rely in turn on assumptions about the composition and temperature profiles through the planet.

Theories of planetary formation can be investigated by comparing the structure of exoplanets with those within the solar system. Another motivation is to estimate the occurrence of Earth-like planets, in terms of mass and composition, and also those that might be habitable for life.

Deductions about planetary structures, i.e. the composition profile, depend on the compressibility of the possible compositions thought to occur. The compressibility is needed over the range of pressures and temperatures occurring within each planet. 
The compressibility is found from the derivative of the pressure-density relation at the appropriate temperature, which can be determined from the equation of state (EOS) for the particular composition of matter of interest. The development of EOS has been driven most by research in weapons (explosives and projectile impacts) (for instance McQueen et al 1970), geophysics (e.g. Ahrens \& Gregson 1964; Ahrens 1966; Alfè et al 2001; Stacey \& Davis 2004; Stixrude et al 2009), and inertial confinement fusion (Lindl 1998). There is a perception that experimental and theoretical methods for determining EOS are not available in regimes necessary to understand the internal structure of planets for pressures between $200 \mathrm{GPa}$ and $10 \mathrm{TPa}$, i.e. from the limit of diamond anvil data to the onset of the Thomas-Fermi-Dirac (TFD) regime (Seager et al 2007; Grasset et al 2009). Some studies (e.g. Seager et al 2007) have considered sub-TFD EOS with care, but it is common practice even when accurate theoretical calculations are available to represent the material with ad hoc functional forms, potentially leading to problems when extrapolating beyond the bounds of the constraining data and in switching over to TFD at high pressures.

Although there is a definite need for more theoretical and experimental studies, appropriate theoretical techniques are just as accurate above $200 \mathrm{GPa}$ as below, and are more than adequate to draw inferences about the structure of exoplanets; moreover, shock and ramp loading experiments can readily explore states up to $\sim 5 \mathrm{TPa}$ and are suitable to test and calibrate EOS. In this paper, we study the validity of electronic structure methods for predicting EOS up to the $o(100) \mathrm{TPa}$ pressures 1 apparently occurring in exoplanets, and the capability of dynamic loading experiments to measure relevant states. We calculate mass-radius relations for several compositions of matter representing different classes of, and layers in, planets, discussing the validity of the EOS used. Finally, we compare the mass-radius relations with representative planets and exoplanets.

\footnotetext{
${ }^{1} \mathrm{~A}$ pressure of $1 \mathrm{TPa}$ is 10 million atmospheres.
} 


\section{Matter at high pressure}

The quasistatic structure of self-gravitating bodies depends on the scalar EOS, which can be described by an appropriate free energy expressed as a function of its natural variables, such as the Helmholtz free energy $f(\rho, T)$, where $\rho$ is mass density and $T$

temperature. In principle, one could consider the composition as a set of explicit parameters in the EOS. In practice, planetary structures are thought to comprise layers in each of which a single composition, or range of compositions, dominates the EOS, such as Fe in the core of rocky planets. Therefore, we consider a separate EOS for each layer.

As with dynamic loading situations, the pressure $p \equiv \partial f /\left.\partial v\right|_{T}$ is the most directly useful aspect of the EOS for calculating and interpreting planetary structures. Almost invariably, a thermodynamically incomplete $\operatorname{EOS}$ is used: $p(\rho, T)$ or $p(\rho, e)$ where $e$ is the specific internal energy. Planetary structures may be constrained to follow a specified temperature profile, in which $p(\rho, T)$ is the more convenient form, or an isentrope, for which $p(\rho, e)$ is convenient since $p=-\partial e /\left.\partial v\right|_{s}$ where $v=1 / \rho$.

In planets, brown dwarfs, and main sequence stars, the EOS is dominated by electrostatic forces and Pauli exclusion among the electrons and ions, rather than by strong-force interactions between the nuclei. In stars, the radiation pressure must be included, and the entropy is much higher, exploring a different region of the EOS.

\subsection{Theory}

In this section, we describe key theoretical methods and approximations used when predicting EOS, and comment on their applicability to states likely to occur in exoplanets.

The relevant EOS of matter can be calculated using electronic structure theory. For a representative set of atoms defining the composition, thermodynamic potentials can 
be calculated as a function of $\rho$ (i.e. system volume) and $T$. Because the mass of the electrons is so much less than that of the nuclei, the state of the electrons can generally be considered with respect to the instantaneous positions of the nuclei - the Born-Oppenheimer approximation. Forces on the nuclei can also be calculated with respect to their positions and the distribution of electrons. The energy of the system can be thought of as comprising the ground state energy for stationary ions, thermal motion of the ions, and thermal excitation of the electrons out of their ground state. In principle, all contributions should be calculated self-consistently. However, for EOS, it is generally a good approximation to partition the free energy into the cold compression curve, thermal motion of the ions, and thermal excitation of the electrons,

$$
f(\rho, T)=f_{c}(\rho)+f_{i}(\rho, T)+f_{e}(\rho, T)
$$

(see Swift et al 2001). To describe the material state and the dynamics of most atoms, the quantum nature of the nuclei can be ignored, allowing their treatment as point-like masses exhibiting Newtonian dynamics. The exception is $\mathrm{H}$, for which the quantum behavior of the proton can be represented as a correction to the potential experienced by the electrons.

The motion of the nuclei can be calculated with respect to time. This technique, known as first-principles molecular dynamics (FPMD) or quantum molecular dynamics (QMD) (Michielsen \& De Raedt 1996), is particularly appropriate for fluids, unknown or ill-defined crystal structures, and multi-species compositions of matter where it is not clear a priori where particular species will be located. In addition to the EOS, FPMD has been used to predict transport properties, including the electric and thermal conductivities (Recoules et al 2009; Hamel et al 2011; Holst et al 2011) and viscosity (Alfè et al 2000a; Clerouin 2002), which are important in understanding the formation and thermal profile of planets as well as the generation of their magnetic fields. FPMD has also been used in studies of the free energy profiles of mixtures to determine possible miscibility gaps which 
could have a large impact on planetary evolution and structure (Morales et al 2009).

For crystalline structures, the motion of the nuclei can be calculated in terms of oscillations about their equilibrium positions, i.e. phonon modes. In principle, phonon modes should be calculated self-consistently with electron excitations; in practice, for most conditions encountered in planets, the ion-thermal motion can be calculated from the phonon density of states at $T=0,2$ and the electron-thermal contribution can often be ignored. $f_{c}$ comprises the ground state energy of the system as a function of compression, $e_{c}(\rho)$, and any configurational entropy that may be associated with different structural polymorphs or the entropy of mixing in an impure system (Kittel \& Kroemer 1980). One may calculate $e_{c}$ by setting up a configuration of nuclei, and finding the ground state of the Hamiltonian of the electrons. The configuration of the nuclei may be altered or relaxed under the net force they experience to find the lowest-energy structure, but they are often held fixed in likely structures. For well-defined crystalline structures, phonon modes can be predicted by calculating the electrostatic forces on the nuclei as each is displaced from equilibrium. Explicit calculation of the phonons is desirable below the Debye temperature, where the zero-point motion of the nuclei and the freezing out of modes may have a significant effect on the EOS (Swift et al 2001).

Thermal excitation of the electrons can be calculated from the band structure, which is the set of eigenstates of the electronic Hamiltonian. For sufficiently high temperatures, the eigenstates must be calculated self-consistently with their population, but accurate calculations can be made to $\sim \mathrm{eV}$ temperatures using the $T=0$ band structure.

The key physics, and thus the source of limitations in the accuracy and validity of the EOS, is the electronic structure calculation. The challenge is in representing the

\footnotetext{
${ }^{2}$ All temperatures here are defined with respect to absolute zero.
} 
multi-fermion nature of the electrons accurately, but with a method that is computationally tractable for real materials. Path integral methods, such as path-integral Monte-Carlo (PIMC), can be used for direct, rigorous calculations of multi-particle states (Militzer 2009). However, these methods are practicable mostly for low- $Z$ materials, and have not been applied systematically (or, generally, at all) to compositions of matter relevant to ice and rocky planets. There is, however, no reason to suspect that these techniques are unsuitable under exoplanet conditions per se.

Although TFD-based electronic structure theory (Salpeter \& Zapolsky 1967) is often regarded as an adequate treatment for pressures over $\sim 10 \mathrm{TPa}$ (for instance Seager et al 2007; Grasset et al 2009), it does not capture the effects of electronic shell structure which are expected at high pressures (Liberman 1979). However, shell effects such as pressure ionization are captured by the electronic structure techniques considered here.

Most theoretical EOS calculations use density functional theory (DFT) (Hohenberg \& Kohn 1964; Kohn \& Sham 1965; Perdew 1992, 1994) and its variants to treat exchange and correlation between the electrons via functionals of the electron density, calibrated to reproduce the same system energy as techniques that treat the electron wavefunctions more directly. DFT functionals are calibrated against calculations of idealized electron gases, which may be performed up to arbitrarily high densities: they should be no less accurate for the relatively modest absolute compressions occurring in the cores of exoplanets than at $p=0$. DFT calculations typically reproduce the observed mass density to within a few percent (for instance Swift et al 2001; Ackland 2002), which is an observable discrepancy when compared with dynamic or quasistatic loading measurements. Calculations can be corrected by adjusting the energy to reproduce the mass density at zero pressure, which is most accurately measured. The resulting EOS - which we have termed 'ab fere initio' to distinguish them from uncorrected ab initio EOS - then typically reproduce high pressure 
measurements to within their uncertainties (Swift et al 2001, 2007).

In many situations, electrons closer to the nuclei are not affected by changes in the compression or temperature, and the states of the other electrons may be calculated more efficiently by subsuming the inner electrons into a modified nuclear potential: the pseudopotential method (Payne et al 1992). Pseudopotential calculations can become inaccurate when the nuclei are compressed sufficiently closely together. The validity can be checked by comparing against all-electron calculations, and may be accurate to severalfold compressions or pressures of terapascals (Morales et al 2009).

With these caveats, pseudopotential techniques and the DFT construct in general are not inherently unsuitable for predicting EOS in the planetary structure regime. However, detailed calculations of many-species compounds are computationally expensive, and predictions of polymorphic structures are sensitive to relatively small inaccuracies in the computational methods. It is highly desirable to compare EOS predictions against experimental measurements.

For the most part, we have used previously-developed EOS based on experimental data or validated electronic structure calculations up to the limit of available data, and blending into TFD predictions at high compressions (Holian 1984). These EOS incorporate thermal effects, allowing us to investigate the sensitivity of planetary structures to temperature profile. For Fe and Fe-Ni, we have compared predictions from such EOS with 3D electronic structure calculations extended to higher pressures than have been reported previously. Previous theoretical studies include very careful treatments of the EOS of Fe up to pressures and temperatures representative of the Earth's core (in particular Wasserman et al 1996; Stixrude \& Cohen 1995; Alfè et al 2000; Sola et al 2009; Belonoshko et al 2008, and references therein), and it is very desirable to perform equivalent studies to higher pressures and temperatures necessary for the study of exoplanets. For our present purposes, it is 
most useful to compare EOS for different compositions of matter constructed according to consistent prescriptions. The use of wide-ranging EOS constructed using optimized algorithms, and wide-ranging sets of electronic structure calculations made using a consistent method that reproduces TFD at extreme compressions, allows us to avoid any reliance on extrapolating using ad hoc functional forms such as Vinet and Birch-Murnaghan for pressure-density relations, which can give unquantified uncertainties outside the range of the fitting data.

\subsection{Dynamic loading experiments}

The canonical experimental technique for studying the properties at matter at high pressure is shock loading, using a variety of methods to induce a shock wave. Shock measurements of EOS are often performed relative to a reference material, but an attraction of shock loading is that experiments can in principle be configured to yield absolute measurements, if the shock is induced by the impact of a projectile with a target of the same material. Indeed, the pressure standards in static compression apparatus such as diamond anvil cells are ultimately calibrated against absolute shock measurements.

Although the time scales in dynamic loading experiments are typically nanoseconds to microseconds, typical equilibration times for electrons and atomic vibrations are much shorter, so inferred states used for testing and calibrating EOS are in thermodynamic equilibrium and thus equivalent to quasistatic compression measurements made in presses such as diamond anvil cells. Indeed, the difference in time scale between dynamic and quasistatic loading is less than the difference between the latter and planetary ages. Although EOS measurements are in thermodynamic equilibrium with respect to a given phase of matter, the time dependence of phase transitions must be considered: phase changes often occur with a significant degree of superpressurization or superheating 
in dynamic loading experiments and indeed in quasistatic loading, compared with the equilibrium phase boundary. The effects of time dependence are also evident as hysteresis in the location of the phase change on loading compared with unloading.

In shock loading, the entropy increases with compression, so the temperature rises faster with compression than it does along an isentrope. The Grüneisen parameter and heat capacity of a material, which may be estimated theoretically or experimentally, can be used to predict the EOS away from the locus of shock states. Measurements along the principal shock Hugoniot have been used in this way for many years to estimate the principal isentrope and the cold curve (Bushman et al 1993). While a direct measurement of a relevant state is preferable, shock-derived EOS are likely to be adequate for exoplanets over a wide range of pressures. Furthermore, if a theoretical EOS is validated by shock experiments, this provides reasonable confidence that the EOS is valid at lower temperatures. A more serious limitation with shock experiments is that, for a given material and starting state, there is a limit to the compression that can be achieved by the passage of a single shock, and therefore a limit to the range of compressions that can be deduced along isentropes. However, this compression (discussed further below for different compositions of matter) is adequate to explore matter into the massive exoplanet regime. Shock measurements have been made to pressures of up to $10 \mathrm{TPa}$ using radiation from underground nuclear explosions (Ragan 1984).

The compression limit can be circumvented through the use of multiple shocks, which induce lower temperatures than a single shock to the same pressure. The ultimate limit for an infinite sequence of infinitesimal shocks is a ramp compression. Ramp compression of condensed matter was first demonstrated using the expanding reaction products from a chemical explosive (Barnes et al 1974), and has subsequently been demonstrated using pulsed magnetic fields at the $\mathrm{Z}$ accelerator (Reisman et al 2001) and high power lasers. 
With lasers, the pulse energy may be used to vaporize a foil which then loads the sample in an analogous way to the chemical reaction products (Edwards et al 2004), the pulse may ablate the sample directly with an intensity history designed to induce a ramp in pressure (Swift \& Johnson 2005), or the sample may be driven from a hohlraum with a power history chosen to control the hohlraum temperature history such that a ramp is induced. Ramp loading using the last variant has been demonstrated to $\sim 1 \mathrm{TPa}$ on the OMEGA laser (Bradley et al 2009), with near-term experiments on Fe planned to reach $2 \mathrm{TPa}$ at the National Ignition Facility (NIF). Experimental techniques have thus been available to develop and test EOS under conditions relevant to exoplanets, and planned developments should provide the first direct measurements of matter under exoplanet core conditions.

\section{Mass-radius relations}

If the rotation rate is not extreme, self-gravitating bodies are close to spherical. Spherical structures are certainly an adequate starting place for studies of exoplanets. The condition for isostatic equilibrium is that the stress induced by pressure variations is balanced by the gravitational acceleration $g(r)$ :

$$
\operatorname{grad} p(r)=-\rho(r) g(r)
$$

For Newtonian gravitation, by Gauss' theorem, $g(r)$ can be expressed in terms of the mass enclosed within a given radius $m(r)$ :

$$
g(r)=\frac{G m(r)}{r^{2}}
$$

so

$$
\frac{d g(r)}{d r}=G\left(\frac{1}{r^{2}} \frac{d m(r)}{d r}-2 \frac{m(r)}{r^{3}}\right) .
$$

$m(r)$ can be calculated simply from the distribution of mass density,

$$
m(r)=4 \pi \int_{0}^{r} r^{\prime 2} \rho\left(r^{\prime}\right) d r^{\prime}
$$


or

$$
\frac{d m(r)}{d r}=4 \pi r^{2} \rho(r)
$$

When the total mass $M$, outer radius $R$, and surface composition are known with reasonable confidence, and the objective is to determine an aspect of the internal structure such as the core radius, the equations can be integrated from the surface inward, starting at the known $p(R)=0$ (hence $\rho(R)$ ) and $g(R)=G M / R^{2}$ (Swift 2009). The internal structure parameter is found as the solution of a shooting problem to ensure that $m(r) \rightarrow 0$ as $r \rightarrow 0$. To calculate generic mass-radius relations for a uniform composition, it is most efficient to start at the center, where $g(0)=0$ and $m(0)=0$, choose the core pressure $p_{c}=p(0)$, and integrate outward until $p=0$. In this way, the mass-radius condition is obtained in terms of $p_{c}$ as an independent parameter: $\{R, M\}\left(p_{c}\right)$.

These solution methods are unwieldy when it is desired to constrain the overall composition as a mixture of components, such as a fixed ratio between Fe, rock, and H/He. In this case, the isostatic structure may be found more conveniently by starting with the desired masses of each component, and solving the system of equations as an elliptic problem. A numerical solution may be found iteratively by taking an approximate solution (which may initially be concentric shells of material at their $p=0$ density) and improving it by calculating the net stress gradient at each radius

$$
\operatorname{grad} \tau=\operatorname{grad} p+\rho g
$$

and

$$
\frac{\partial p}{\partial r} \simeq \frac{\partial p}{\partial \rho} \frac{\partial \rho}{\partial r}
$$

(ignoring the relatively slow change of $g$ with deformation, which is valid near equilibrium), and hence estimating a change in radius for each shell such that the net pressure is zero. The radial variation was represented discretely by values at a finite series of radii. Multiple 
iterations are necessary to allow the effect of a net pressure at some radius to be balanced through equilibration of the whole structure, but standard techniques may be used for solving elliptic equations efficiently, such as successive over-relaxation (Press et al 1989). A series of solutions describing a mass-radius relation can be found efficiently by working upward in mass, and increasing the density at each radius by a constant factor, then calculating the modified equilibrium structure. We verified that this method of solution gave the same result as integration from the center to the surface, by comparison with structures comprising pure Fe.

To close the system of equations, a $p(\rho)$ relation is needed for the material. The choice of $p(\rho)$ relation implies a choice for the entropy or temperature profile of the body. In the present study we usually chose an isentrope passing through a reasonable surface state, such as STP for rock or metal, or a few atmospheres pressure and cryogenic temperatures for compositions that are gaseous at STP. Isentropes were calculated by numerical integration of the relation

$$
\left.\frac{\partial e}{\partial v}\right|_{s}=-p(\rho, e)
$$

using a procedure valid for EOS of arbitrary form (Swift 2008). For a few sample compositions, structures were calculated for $p(\rho)$ along the cold curve. The precision of $p(\rho)$ curves necessary to be useful for exoplanet structures is lower than for solar system planets, and the difference in structures between the STP isentrope and cold curve was found to be negligible over the range of pressures considered, as shown below.

Mass-radius relations were calculated for a series of compositions of matter relevant to (exo)planets, using as a baseline EOS developed from and for shock wave applications, in particular the SESAME library (Holian 1984), for which the constituent assumptions and calibrations are reasonably well documented. SESAME EOS are tabulated functions $\{p, e\}(\rho, T)$. Most SESAME EOS are tabulated over a wide enough range of states 
to be valid (at least in principle, to some finite precision) for massive exoplanets and star formation. They were constructed to incorporate experimental data, mainly shock measurements, where available. Experimental measurements not used in the construction of the EOS, including shock states, release from shock, and ramp compression, are frequently compared against the SESAME EOS, so there is a relatively rich literature on experimental validation. Many of the EOS were constructed using the same theoretical and modeling approaches, so conclusions drawn from the use of one EOS are more likely to apply to other materials. When these EOS are compared with subsequent shock or ramp measurements at higher pressures, it is quite usual to find significant differences, i.e. inaccuracies in the EOS, but they are usually at the level of a few times the experimental uncertainty (for instance Hicks et al 2005; Knudson \& Desjarlais 2009), which would not have much effect on the mass-radius relation.

For all undifferentiated compositions, the low-mass limit behaves as

$$
M=\frac{4}{3} \pi R^{3} \rho_{0}
$$

and deviates as matter within the planet is compressed gravitationally above its zeropressure density $\rho_{0}$. The deviations become substantial as the central pressure approaches the bulk modulus. As the mass increases further, an increasing proportion is compressed to significantly higher density, until eventually any increase in mass is accommodated entirely by density increase, leading to a maximum in radius.

\subsection{Fe and Fe-Ni}

For Fe, which is the dominant component of interest at the highest pressures, we are developing EOS better optimized to exoplanet core states, to complement NIF experiments (Swift 2010). Thermodynamically complete versions of these EOS were not available for 
this study: only the cold curves. The effect of temperature was assessed by comparing structures calculated with $p(\rho)$ along an isentrope compared with the cold curve, for thermodynamically complete Fe EOS, and was found to be negligible, in agreement with previous work using a simplified thermal contribution (Seager et al 2007). The mass-radius relation derived from our new Fe cold curve, which was calculated with DFT using the local density approximation and Troullier-Martins pseudopotentials (Troullier \& Martin 1991), agreed well with the relations from SESAME EOS 2140 (Barnes \& Rood 1973) and 2150 (Kerley 1993) (Fig. 1). Fe ${ }_{3} \mathrm{Ni}$ was considered as a representative Fe-Ni composition, calculated using the same DFT method as for previous studies of intermetallic compounds (Swift et al 2005, 2007). The pseudopotential for Ni was used previously for constructing EOS for NiAl (Swift et al 2007). Comparing $p(\rho)$ relations, $\mathrm{Fe}_{3} \mathrm{Ni}$ was significantly softer than Fe or a segregated Fe-Ni mixture of the same composition, but the mass-radius relations did not differ significantly. The mass-radius relation was also calculated for the principal isentrope from the linear Grüneisen EOS found previously to perform well for rocky planets (Swift 2009); it deviated from the other Fe relations for masses above approximately twice that of the Earth $\left(\mathrm{M}_{\mathrm{E}}\right)$, demonstrating the inaccuracy of mass-radius relations deduced from EOS extrapolated from low-pressure measurements. Mass-radius relations deduced from the DFT cold curves were very similar to those from the SESAME EOS up to around $1 \mathrm{R}_{\mathrm{E}}$ or $3 \mathrm{M}_{\mathrm{E}}(\sim 1 \mathrm{TPa})$, above which the properties calculated with DFT became progressively softer. This observation highlights the importance of ramp-loading experiments to several terapascals. With the exception of the linear Grüneisen EOS, these relations are similar, but not identical, to relations used recently in the interpretation of exoplanet CoRoT-7b (Léger et al 2009) and for Fe mass-radius relations (Fortney et al 2007; Valencia et al 2010). Interestingly, the relation by Valencia et al follows the linear Grüneisen curve up to around $2 \mathrm{M}_{\mathrm{E}}$, then approaches the $T=0$ DFT relations above $10 \mathrm{M}_{\mathrm{E}}$; the Fortney et al relation is stiffer (larger radius) up to around $1 \mathrm{M}_{\mathrm{E}}$, then follows 
the relation for SESAME 2150 very closely. The mass-radius relations derived from the SESAME EOS were denser above $1 \mathrm{M}_{\mathrm{E}}$. (Figs 1 and 2 ,)

For Fe, the maximum shock compression deduced from the SESAME EOS was a factor of around 5.2, calculated using a solution method valid for general forms of the EOS (Swift) 2008). At the same mass density on the principal isentrope, the pressure is approximately $11 \mathrm{TPa}$. Thus single shock experiments, corrected appropriately for thermal effects, can plausibly explore planetary pressures to this regime. Impact-induced shock experiments, with the projectile driven by gas, chemical propellant, or high explosive, have been performed on Fe to around $1 \mathrm{TPa}$ by several groups (van Thiel 1966). At higher pressures, data are very rare. Nuclear-driven shock impedance mismatch measurements (Ragan 1984) reached a pressure which we estimate to be $4.57 \pm 0.26 \mathrm{TPa}$. Further measurements above $1 \mathrm{TPa}$ are desirable.

\subsection{Rocks}

High quality theoretical and experimental studies have been performed for mantle constituents such as $\mathrm{MgSiO}_{3}$ and $\mathrm{SiO}_{2}$ up to pressures around $150 \mathrm{GPa}$ relevant to Earth's core-mantle boundary, and the effect of Fe ions (see Drummond \& Ackland 2002; Caracas \& Cohen 2008; Stixrude \& Lithgow-Bertelloni 2010; Wentzcovitch et al 2010; Stixrude \& Lithgow-Bertelloni 2011). As compositions representative of the variations in rocks, mass-radius relations were calculated for $\mathrm{SiO}_{2}, \mathrm{MgO}$, and basalt. $\mathrm{SiO}_{2}$ was represented by SESAME EOS 7383 (Johnson \& Lyon 1984). MgO was represented by SESAME EOS 7460 (Barnes \& Lyon 1988a), and also by a DFT-derived two phase EOS including quasiharmonic phonons (Drummond \& Ackland 2002; Luo et al 2004), but extending only to $\sim 0.4 \mathrm{TPa}$. The mass-radius relations for the different $\mathrm{MgO}$ models were indistinguishable, demonstrating insensitivity of the mass-radius relation to the inclusion of 


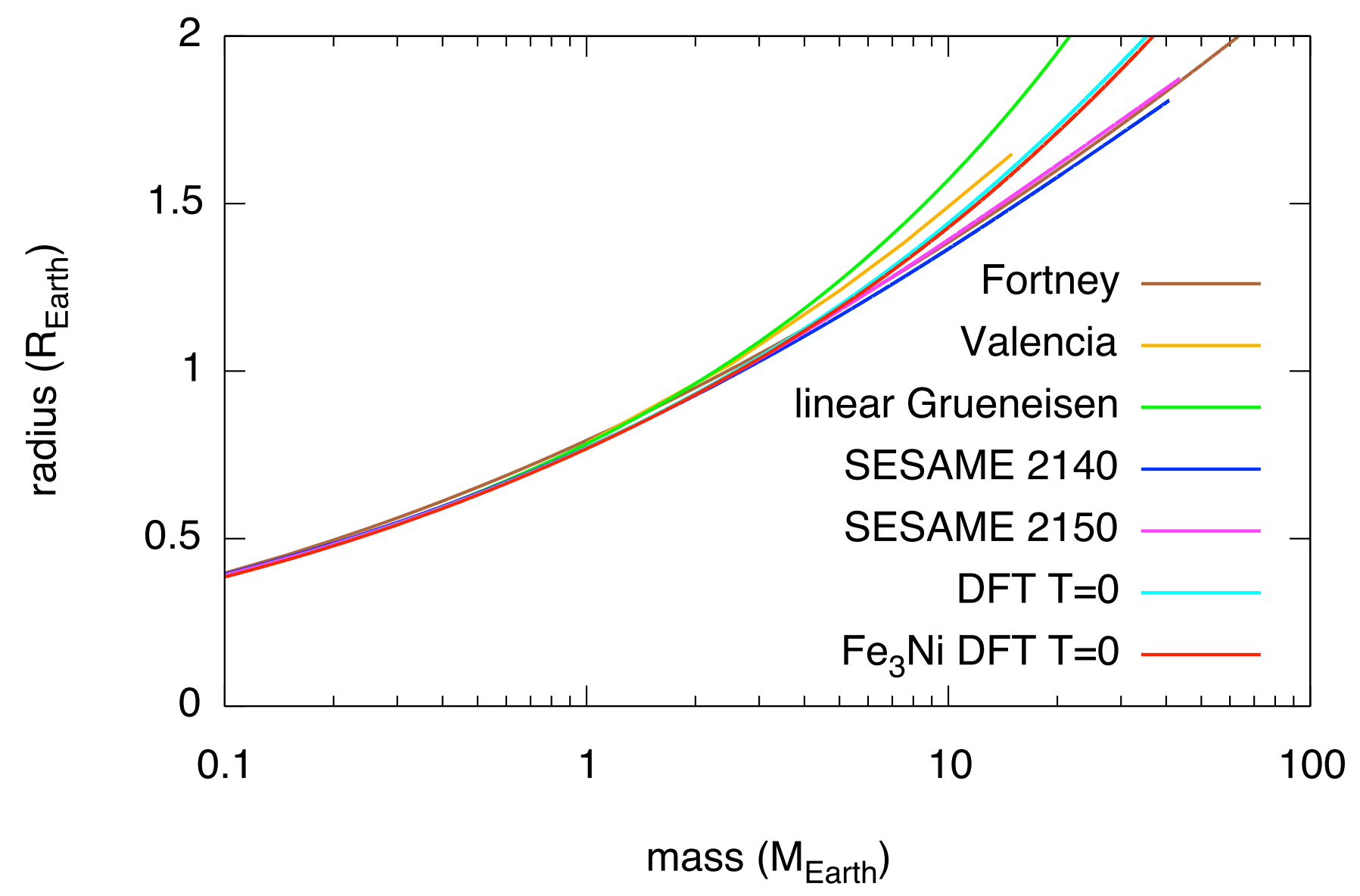

Fig. 1.- Mass-radius relations calculated for different Fe EOS and for $\mathrm{Fe}_{3} \mathrm{Ni}$. 


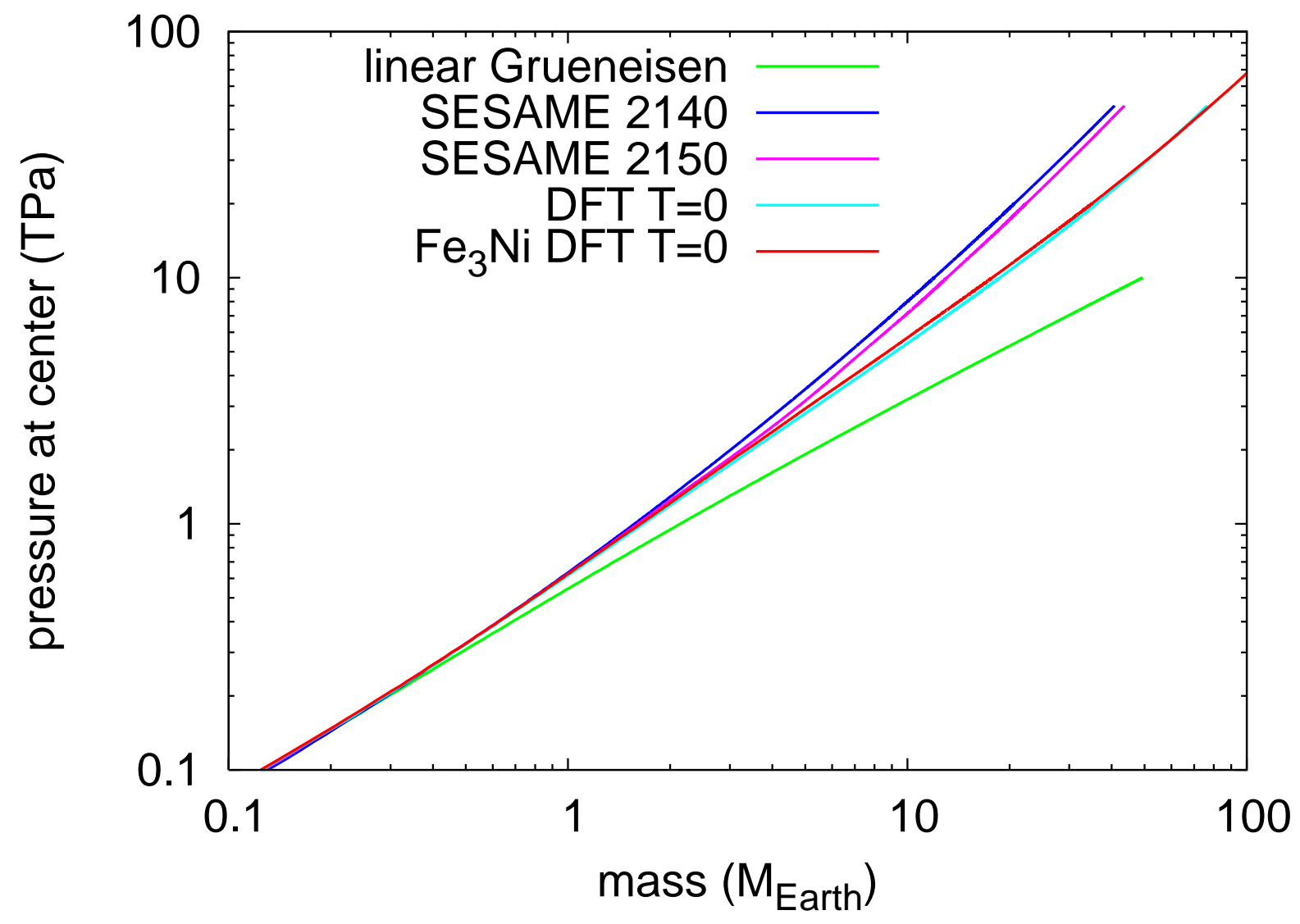

Fig. 2.- Predicted variation of core pressure with mass for different Fe EOS and for $\mathrm{Fe}_{3} \mathrm{Ni}$. 
phase transitions, for current exoplanet applications. Basalt was represented by SESAME EOS 7530 (Barnes \& Lyon 1988b). 'Basalt' refers to the composition (by number of atoms: $60.11 \%$ O, $18.26 \%$ Si, $5.96 \%$ Al, $4.01 \%$ Ca, 3.48\% Fe, 3.39\% Mg, 2.18\% H, 1.56\% Na, 0.55\% Ti, $0.38 \% \mathrm{~K}, 0.07 \% \mathrm{P}, 0.06 \% \mathrm{Mn}$ ) and initial state: the EOS was derived from shock data including phase changes, and was blended into TFD predictions at high pressures. We also compared the linear Grüneisen EOS for basalt found previously to give better than expected structures for the rocky planets (Swift 2009); its mass-radius relation was similar to that of the SESAME EOS to several tenths of a terapascal, and then diverged significantly. The mass-radius and pressure-radius relations were similar but distinguishable on scales extending to exoplanets, giving an indication of the possible uncertainty in inferences about rocky planet structure in the absence of constraints on the composition: the mass-radius relation does show sensitivity to the composition, but the sensitivity is small compared to current uncertainties in exoplanet observables. Recent mass-radius calculations by Valencia et al (2010) for Mg-silicate planets were almost indistinguishable from the $\mathrm{MgO}$ relation; slightly older calculations by Fortney et al (2007) using a linear mixture of $38 \%$ $\mathrm{SiO}_{2}, 25 \% \mathrm{MgO}, 25 \% \mathrm{FeS}$, and $12 \% \mathrm{FeO}$ followed our calculation for basalt (SESAME) very closely to several tens of $\mathrm{M}_{\mathrm{E}}$. (Figs 3 and 4 .)

\subsection{Ices}

Ab initio treatments of mixtures of molecules of low molecular weight, usually called ices (regardless of being in the solid or fluid state), are complicated and computationally expensive because of the possibility of segregation of species and the formation of a wide variety of chemical species. At issue here is the uncertainty of the equilibrium concentration of the different molecules. Such calculations have been performed for relatively small numbers of thermodynamic states (Chau et al 2011), and will in future allow wide-ranging 


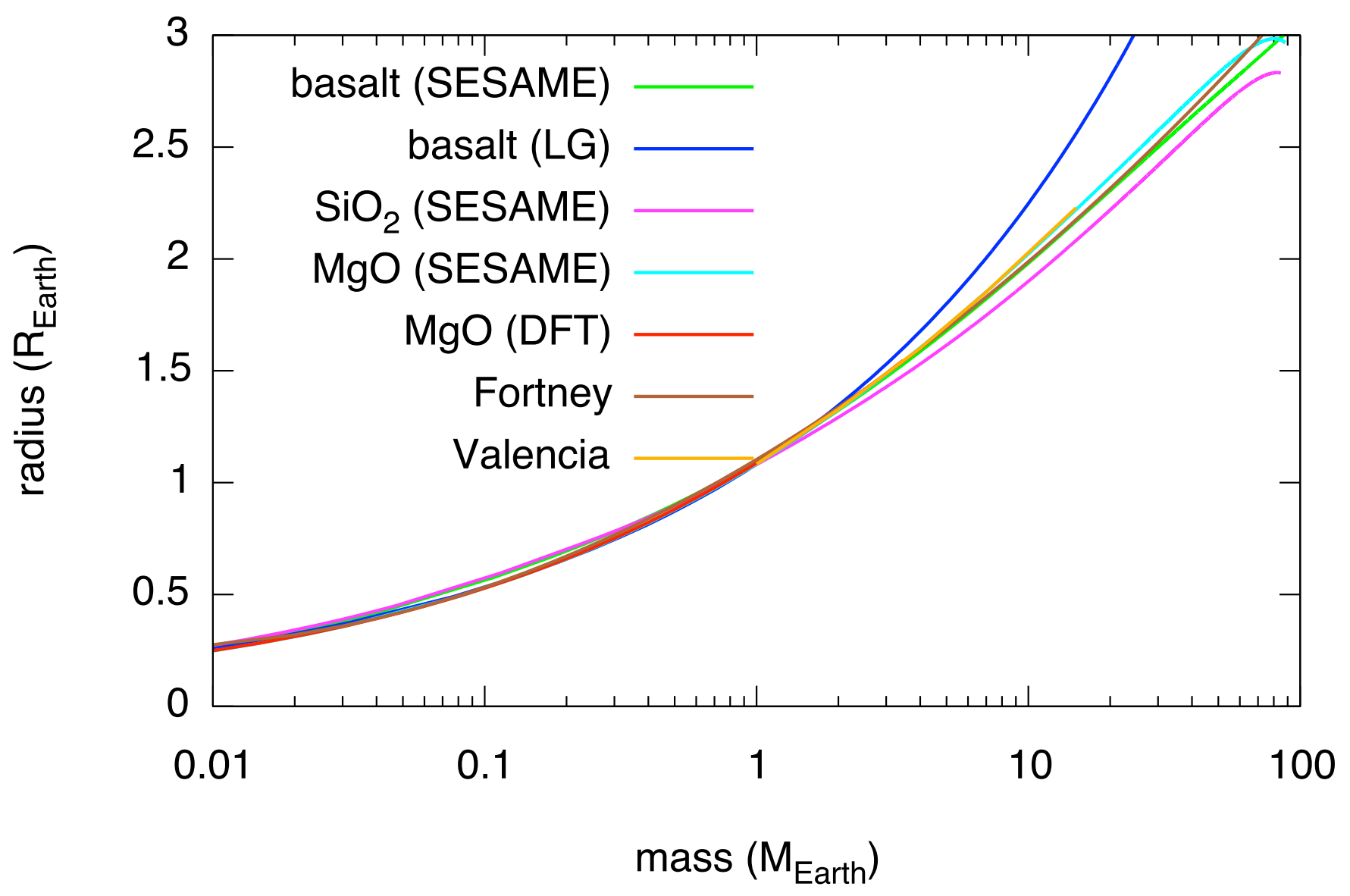

Fig. 3.- Mass-radius relations calculated for $\mathrm{SiO}_{2}, \mathrm{MgO}$, and basalt, including a simplified, linear Grüneisen EOS (LG), and comparing with previous calculations (Fortney et al 2007; Valencia et al 2010). 


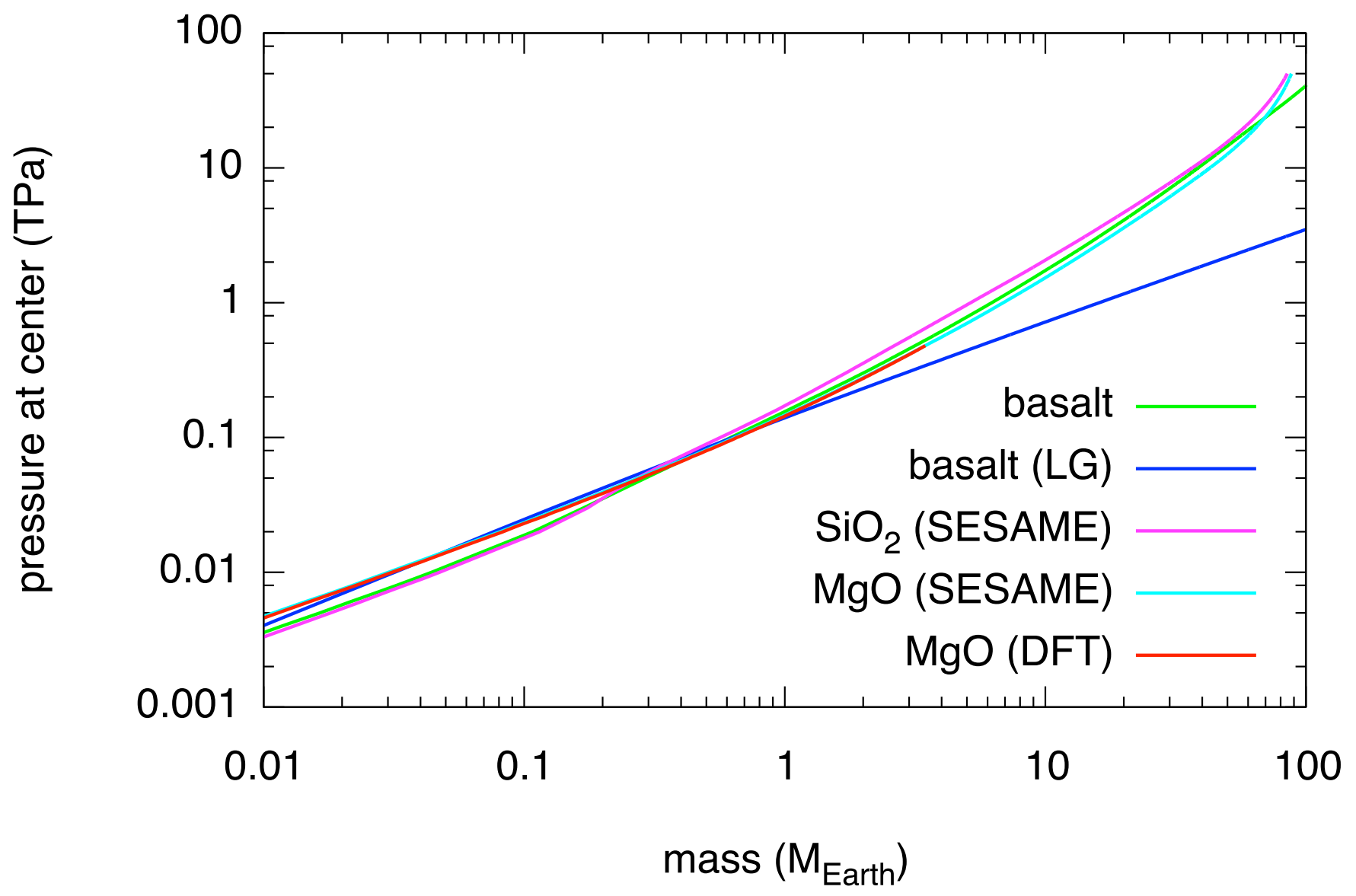

Fig. 4.- Predicted variation of core pressure with mass for $\mathrm{SiO}_{2}, \mathrm{MgO}$, and basalt, including a simplified, linear Grüneisen EOS (LG). 
EOS to be predicted as a function of composition. A complementary approach is to calculate the equilibrium chemical composition given the thermodynamic state and atomic composition, using measured or estimated thermodynamic potentials for each possible chemical specie. Chemical equilibrium calculations have been used to predict the EOS of the reaction products of chemical explosives up to temperatures of several hundred gigapascals and temperatures of $\sim \mathrm{eV}$, including many species in common with planetary ices (Bastea et al 2007; Mulford \& Swift 2006). However, the thermodynamic potentials are not necessarily well-constrained for the hot, compressed states occurring within exoplanets.

As compositions representative of the variations in ice giants, mass-radius relations were calculated for $\mathrm{H}_{2} \mathrm{O}, \mathrm{NH}_{3}$, and $\mathrm{CH}_{4}$, for isentropes chosen to pass through a condensed phase so that the planet surface could be defined as zero pressure. The calculated radius is thus a lower bound for planets with a thick atmosphere, as is likely to be the case for those orbiting close to their star. Several EOS were available for $\mathrm{H}_{2} \mathrm{O}$; SESAME EOS 7154 was the most appropriate for these pressures (Johnson \& Lyon 1990). The STP isentrope was used. The only SESAME EOS available for $\mathrm{NH}_{3}$ was 5520 (Haar \& Gallagher 1978), fitted to National Bureau of Standards (NBS) gas phase data, and was tabulated to a maximum mass density of $0.765 \mathrm{~g} \mathrm{~cm}^{-3}$, which is barely greater than typical for liquid ammonia. An empirical linear Grüneisen EOS was constructed from shock wave data on liquid ammonia at an initial temperature of $203 \mathrm{~K}$ (Swift 2011), using an approximate treatment for off-Hugoniot states. The corresponding mass-radius calculation should be regarded with caution as it is not constrained by experimental data for pressures above a few tens of gigapascals. The mass-radius relation and central pressure were in reasonable agreement with predictions using SESAME EOS 5520 up to a mass $\sim 0.1 M_{E}$ above which the isentrope from the tabular EOS became unusable. For $\mathrm{CH}_{4}$, three SESAME EOS were available: 5500 and 5501 (Kerley 1980), both tabulated to $2.5 \mathrm{~g} \mathrm{~cm}^{-3}$ and respectively with a Maxwell construction and van der Waals loops in the liquid-vapor region; and 
5502 (Johnson 1984), tabulated to $0.47 \mathrm{~g} \mathrm{~cm}^{-3}$ and based on NBS gas phase data. The mass-radius relation was calculated for 5500, which is the most relevant for planetary structures, for an isentrope passing through $70 \mathrm{~K}$ at zero pressure. The difference between $\mathrm{H}_{2} \mathrm{O}$ and $\mathrm{CH}_{4}$ was considerable: it is difficult to infer a composition for an ice giant from the mass-radius measurement alone to a factor $\sim 2$ in mass or $\sim 25 \%$ in radius, without further constraints on the composition. (Figs 5 and 6.)

\subsection{Hydrogen}

Jovian planets are thought to be composed predominantly of a mixture of $\mathrm{H}$ with approximately $5-10 \%$ He by number of atoms.

$\mathrm{H}$ is theoretically interesting because quantum mechanical uncertainty in the position of the proton is more important than for other elements, and may affect the EOS. Significant work has been devoted to predicting the EOS of $\mathrm{H}$ and $\mathrm{H} / \mathrm{He}$ mixtures (Saumon \& Chabrier) 1992; Delaney et al 2006; Morales et al 2009). These H/He mixtures are challenging because even the relatively small amount of helium involved is thought to greatly impact the EOS. Specifically, the insulator-to-metal transition that occurs at o(100) GPa pressures in pure H (Morales et al 2010; Lorenzen et al 2010) is thought to be suppressed to even higher pressures and temperatures with the inclusion of trace amounts of He (Hensel 1999). Furthermore, helium is thought to segregate (i.e. form droplets) in the metallic region of the planet(Morales et al 2009), where the resulting He rain is thought to produce heat as the droplets fall down the gravitational well. This effect could have a disproportionately large effect on the mean EOS and on the differentiation of a planet by gravitational separation.

We did not find a $\mathrm{H} / \mathrm{He}$ EOS of similar pedigree to the other EOS above, so the mass-radius relation was calculated for pure H (SESAME EOS 5251: Albers \& Johnson 


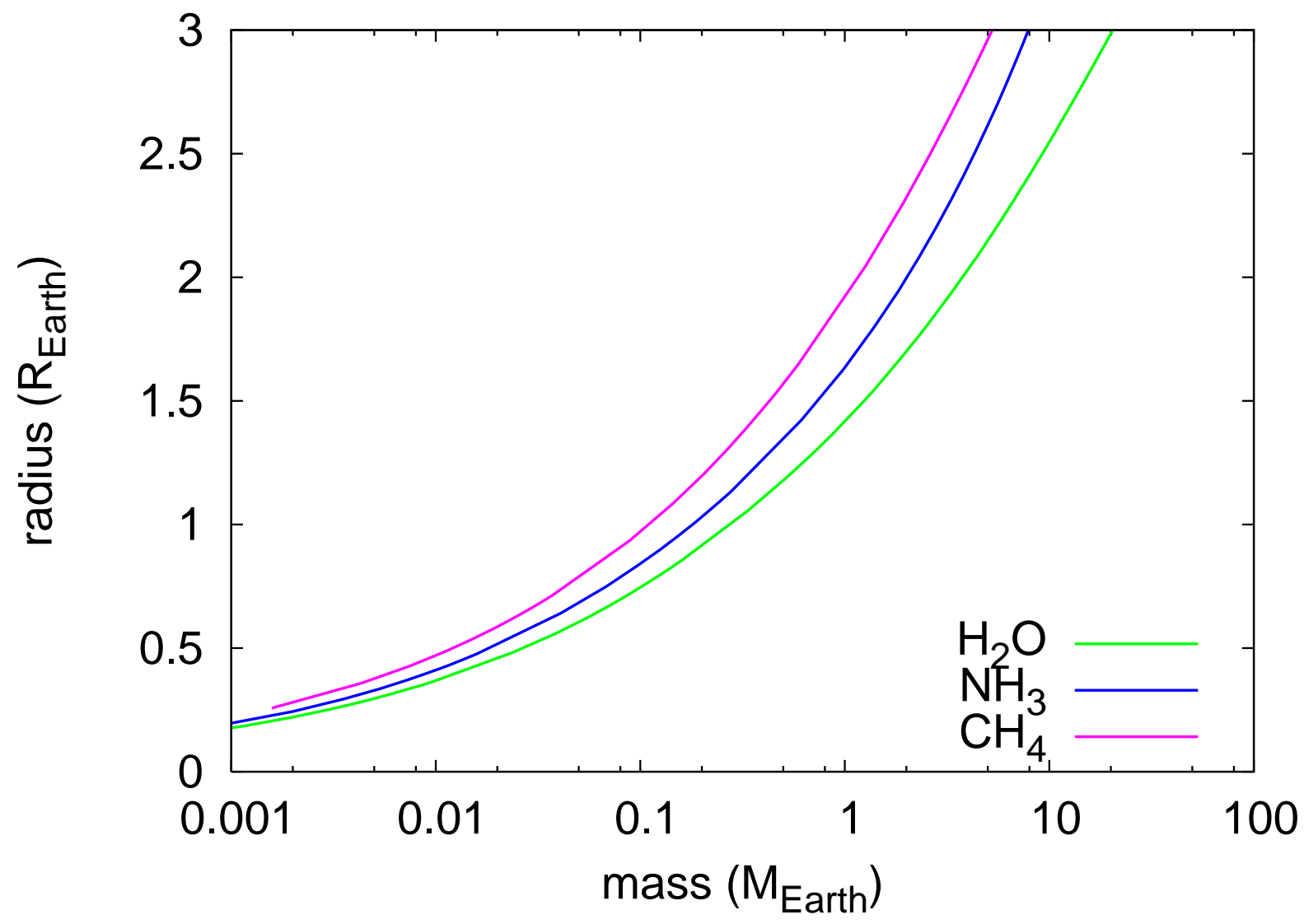

Fig. 5.- Mass-radius relations calculated for $\mathrm{H}_{2} \mathrm{O}, \mathrm{NH}_{3}$, and $\mathrm{CH}_{4}$ ices. 


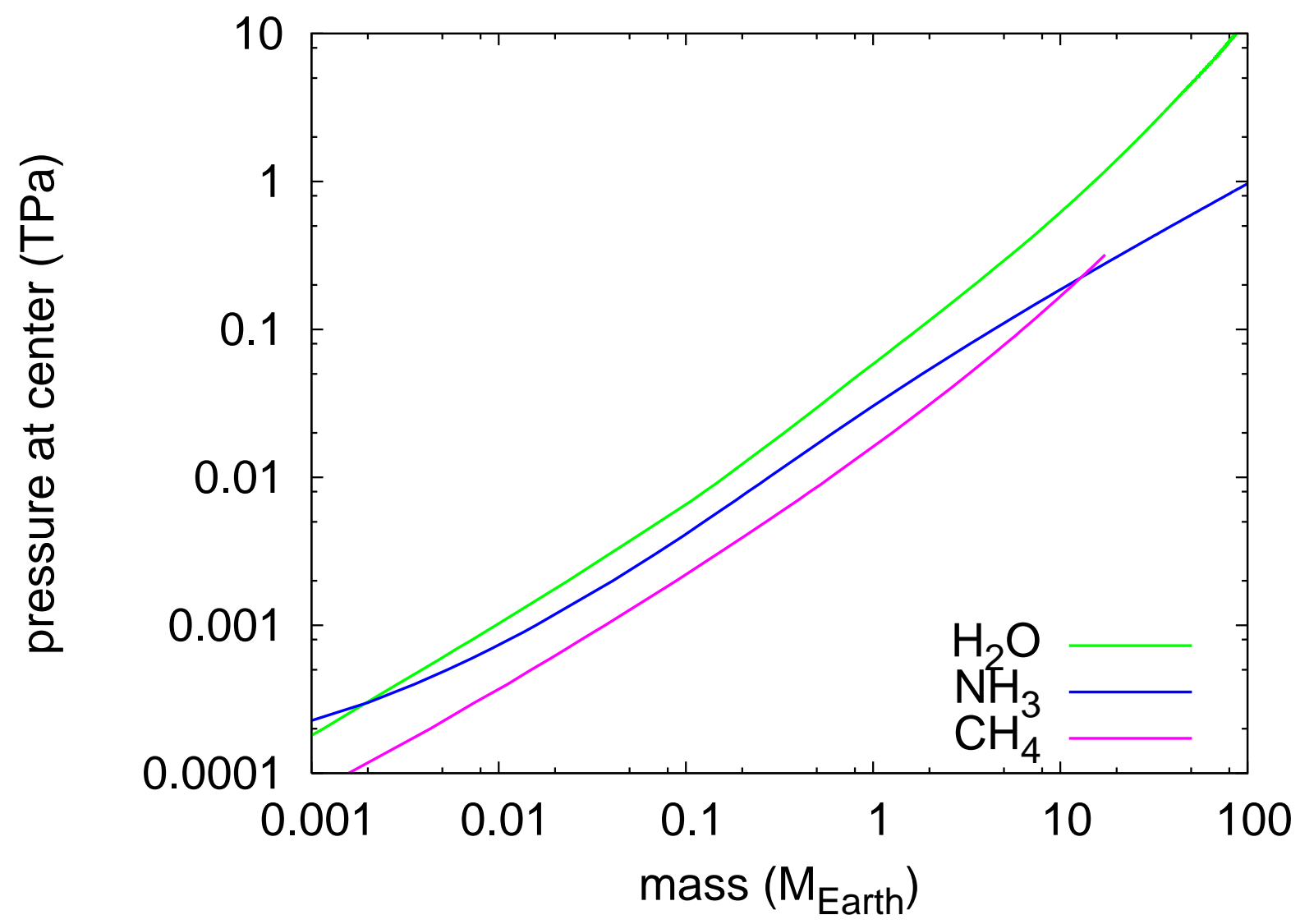

Fig. 6.- Predicted variation of core pressure with mass for $\mathrm{H}_{2} \mathrm{O}, \mathrm{NH}_{3}$, and $\mathrm{CH}_{4}$ ices. The crossing of the $\mathrm{CH}_{4}$ by the $\mathrm{NH}_{3}$ curve is at a mass density much greater than the shock data used to construct the $\mathrm{NH}_{3}$ EOS, and should not be regarded as a meaningful prediction. The crossing of the $\mathrm{H}_{2} \mathrm{O}$ and $\mathrm{NH}_{3}$ curves is in a regime where the EOS should be adequately accurate. 
(1982)) as a limiting case of low density. This EOS was isotopically scaled from SESAME EOS 5263 for $\mathrm{D}_{2}$, which is wide-ranging and includes dissociation and ionization (Kerley 1972), and has been found to give good agreement with static and shock compression data. As with the ices, the isentrope was chosen such that a well-defined planetary surface was calculated, and is thus a lower bound on the radius of planets with a thick atmosphere. For the EOS used, the isentrope passed through $10 \mathrm{~K}$ at zero pressure, and exhibited a kink at around $100 \mathrm{M}_{\mathrm{E}}$ caused by metallization at around $200 \mathrm{GPa}$. Interestingly, the resulting mass-radius relation is quite close to the TFD prediction (Seager et al 2007). Our calculation has a maximum in radius, $79000 \mathrm{~km}$ at a mass of $11 \times 10^{16} \mathrm{~kg}$. This result is slightly higher in mass and lower in radius than the TFD prediction. (Figs 7 and 8, )

\subsection{Fe/basalt}

Mass-radius relations were calculated for differentiated two-component planets comprising an Fe core and a basalt (composition) mantle, with fixed mass ratios, using the elliptic method of solution. Fe and basalt were represented by SESAME EOS 2150 and 7530 respectively. Because of its low density, the addition of an outer basalt layer gave a disproportionate change in the radius, to a greater extent than indicated in previous work (Seager et al 2007). (Fig. 9.)

\section{Application to exoplanets}

For comparative purposes, we chose to represent each type of material by a single mass-radius curve corresponding to a single EOS: Fe-Ni by the electronic structure cold curve for $\mathrm{Fe}_{3} \mathrm{Ni}$ (which is similar to the Fe SESAME 2150 curve but extends to higher pressure), rock by SESAME 5530 for basalt, ice by SESAME 7154 for $\mathrm{H}_{2} \mathrm{O}$, and $\mathrm{H} / \mathrm{He}$ by 


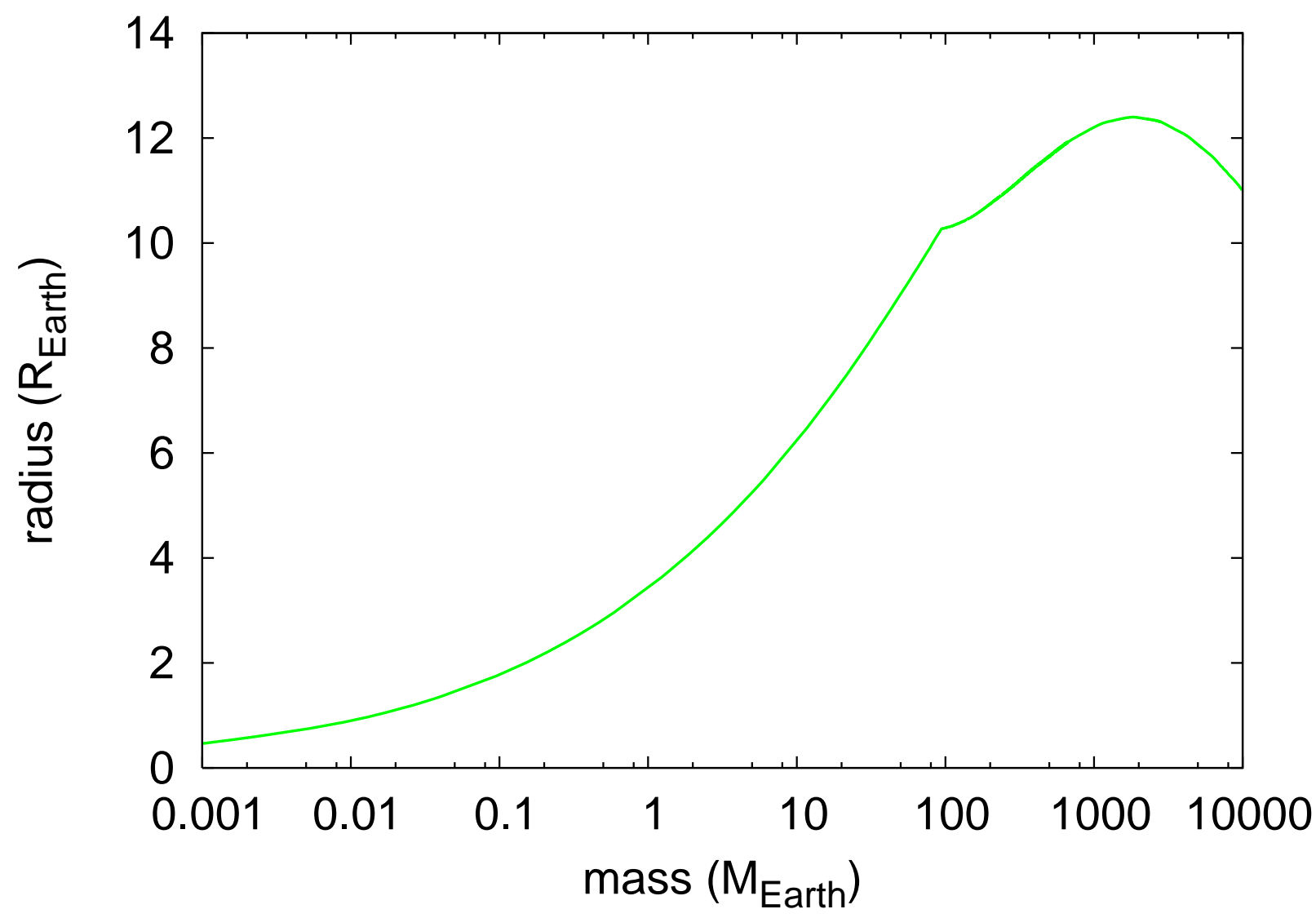

Fig. 7.- Mass-radius relation calculated for $\mathrm{H}$. 


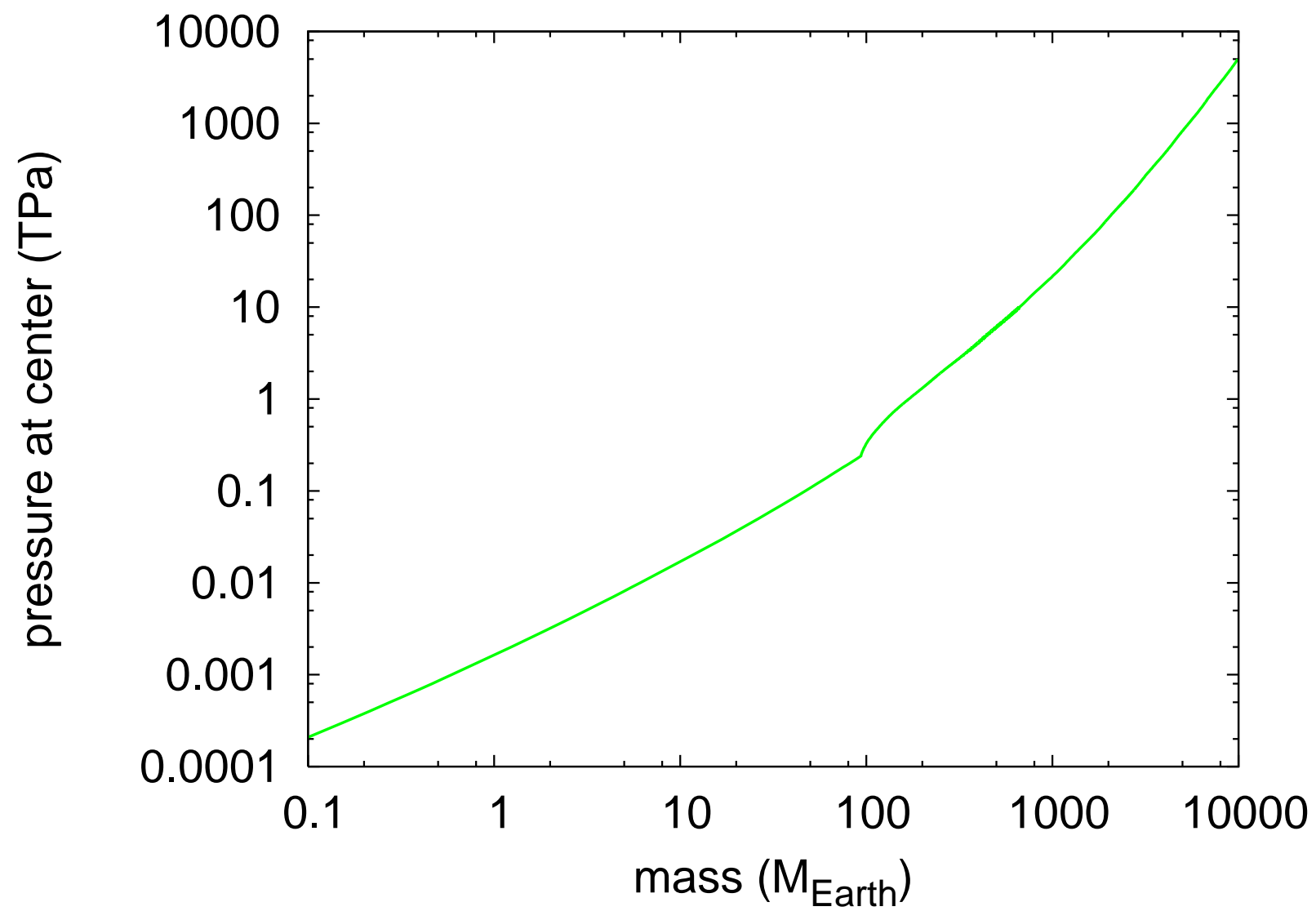

Fig. 8.- Predicted variation of core pressure with mass for $\mathrm{H}$. 


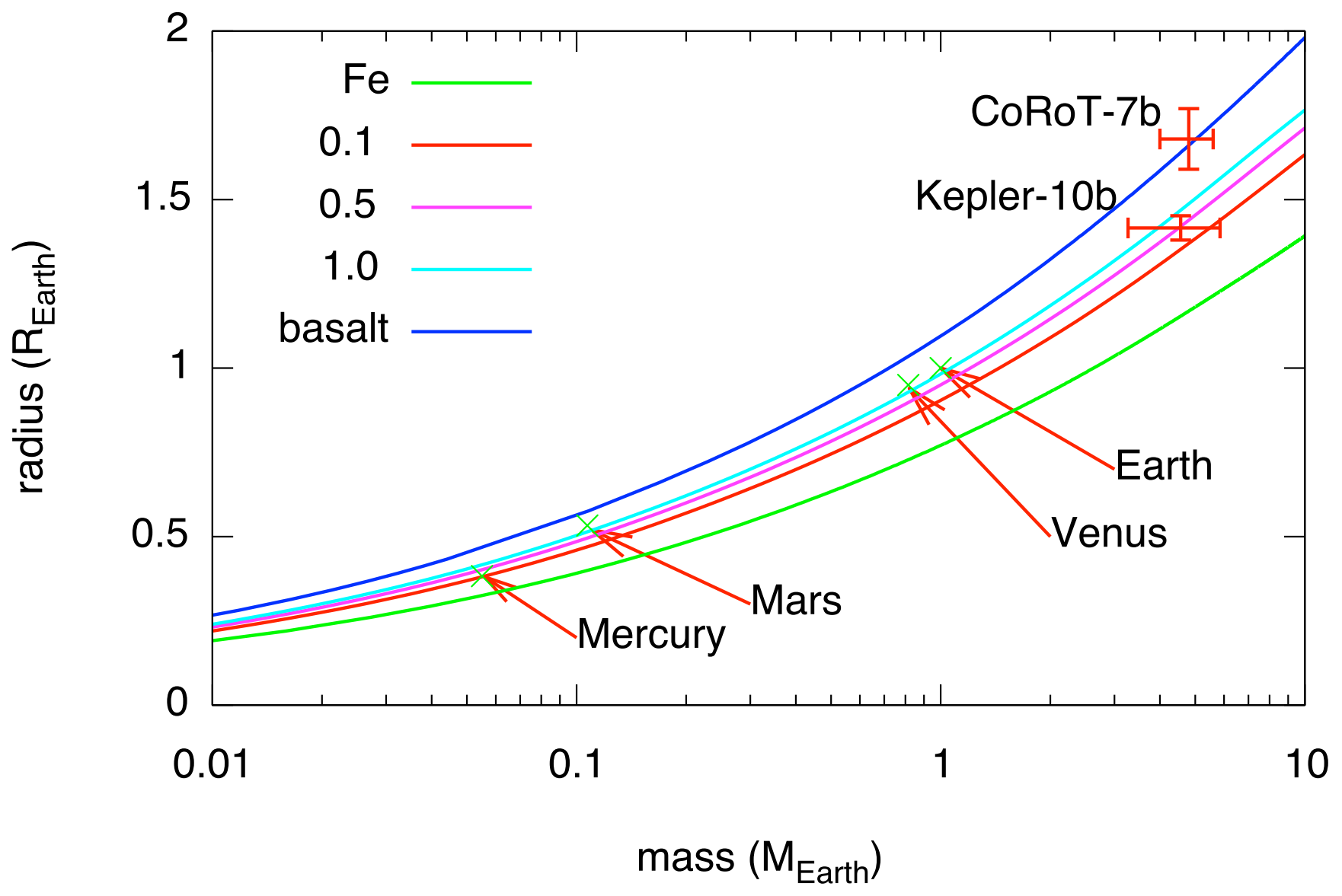

Fig. 9.- Mass-radius relations calculated for differentiated Fe/basalt planets, in terms of the ratio of basalt to Fe. 
SESAME 5251 for H. Plotting the planets of the solar system, they fall in the expected places with respect to the curves: the rocky planets between Fe-Ni and rock with Mercury closest to Fe-Ni and Mars closest to rock, Jupiter and Saturn close to the H/He curve, and Uranus and Neptune close to the $\mathrm{CH}_{4}$ curve. Minor planets Pluto and Eris are also shown, lying on the icy side of the basalt curve. We also compare exoplanets at extremes of size and mass: 'super-Earths' Kepler-10b (Batalha et al 2011), CoRoT-7b (Léger et al 2009), and GJ 1214b (Charbonneau et al 2009) and the 'super-Jupiters' HAT-P-2b (Loeillet et al 2008) and CoRoT-3b (Deleuil et al 2008), whose core pressures are representative of states it would be useful to explore in future experiments on material properties, and the anomalously large CoRoT-2b. (Figs 10 and 11; exoplanet parameters from Schneider (2011).)

Measurements of exoplanet Kepler-10b constrain its radius to $4.56_{-1.29}^{+1.17} M_{E}$ and mass to $1.416_{-0.036}^{+0.033} R_{E}$. The nominal value lies on our mass-radius relation for Fe-basalt differentiated planets with $2 / 3$ of the total mass in the core. With this structure, the central pressure would be $2.5_{-0.3}^{+0.4} \mathrm{TPa}$. Considering two-layer differentiated structures at the $1 \sigma$ level, the planet could have a core mass fraction between $25 \%$ and $95 \%$, with central pressure between about 1.5 and $3.7 \mathrm{TPa}$.

Measurements of exoplanet CoRoT-7b constrain its radius to $1.68 \pm 0.09 \mathrm{R}_{\mathrm{E}}$ and mass to $4.8 \pm 0.8 \mathrm{M}_{\mathrm{E}}$ (Léger et al 2009), though other researchers have deduced different, and inconsistent, masses from the same Doppler shift data (Hatzes et al 2010; Pont et al 2011; Ferraz-Mello et al 2011). Given the current discrepancy in mass deduced by different groups, the validity of deduced compositions and central pressures is unclear, but it is informative to assess the values and uncertainties derived from the quoted uncertainties in mass and radius. The nominal value by Léger et al (2009) lies very close to our mass-radius relation for basalt, for which the central pressure would be $0.75_{-0.12}^{+0.14} \mathrm{TPa}$. Considering 


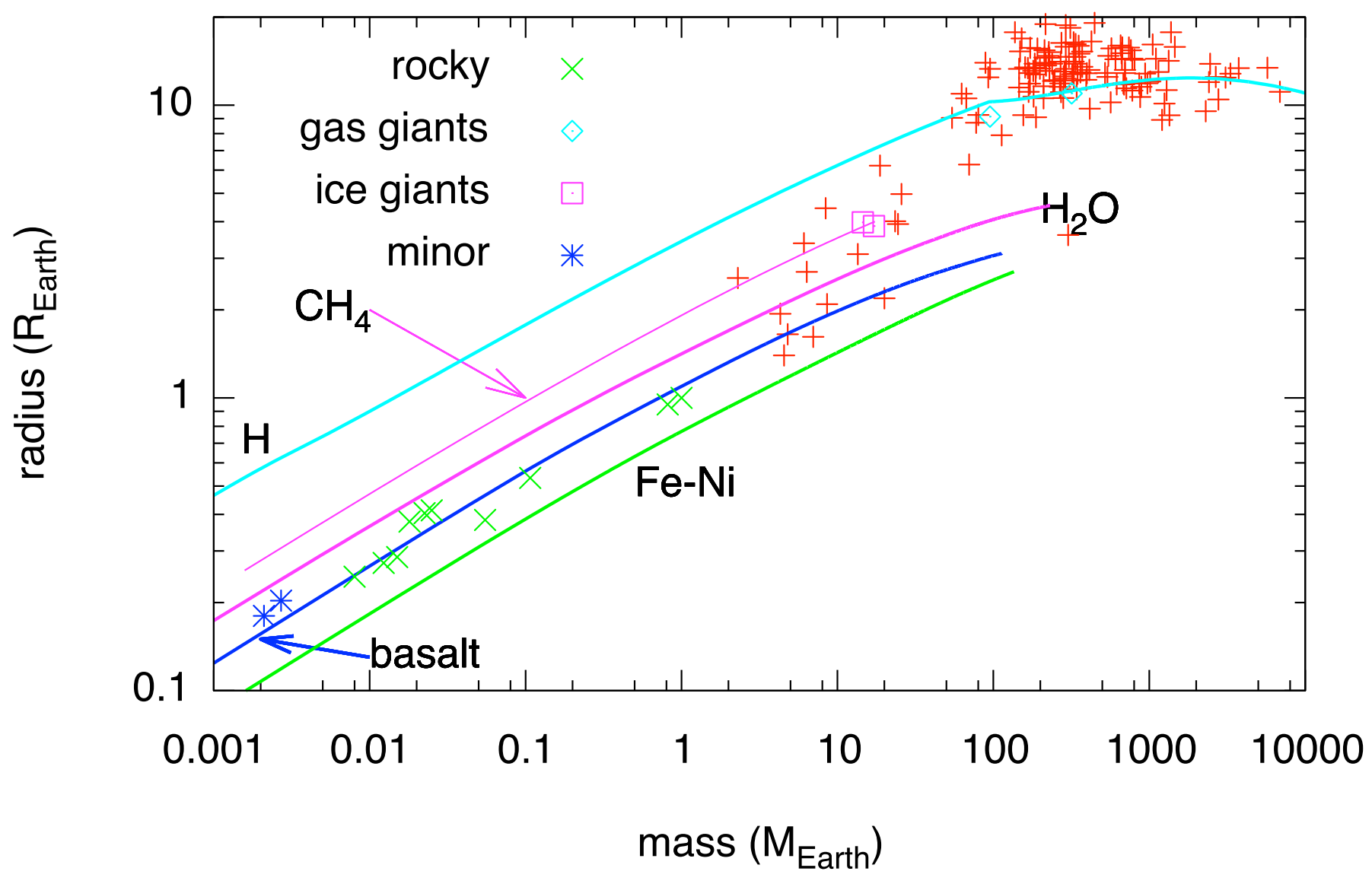

Fig. 10.- Mass-radius relations for different classes of material, compared with planets, moons, and exoplanets (red crosses). 


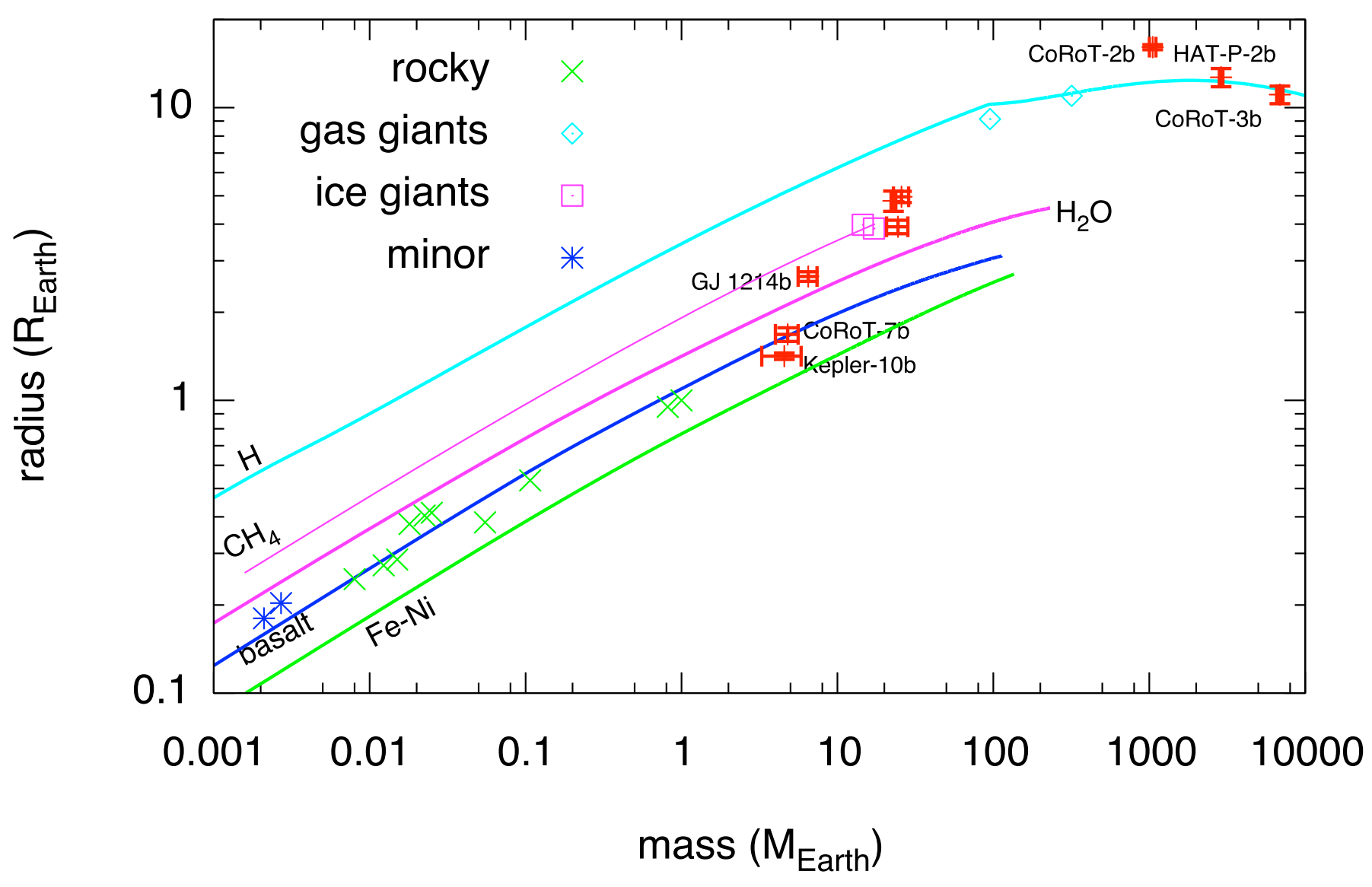

Fig. 11.- Mass-radius relations for different classes of material, compared with selected exoplanets. 
two-layer differentiated structures at the $1 \sigma$ level, the planet could be Fe/rock with a core of radius up to $4500 \mathrm{~km}$, or rock/ice with a surface layer of (for example) $\mathrm{H}_{2} \mathrm{O}$ up to $2500 \mathrm{~km}$ thick. The central pressure is calculated to increase rapidly with Fe, giving $0.8_{-0.1}^{+1.2} \mathrm{TPa}$. For three-layer differentiated structures, the ice layer would be thicker as the core size increased. The uncertainty in deduced composition, core radius, and central pressure is nonlinear with respect to the uncertainty in mass and radius. Measurements of Doppler shift in the stellar spectrum and dimming in the stellar brightness are essentially independent, so we calculate uncertainty contours in radius and central pressure and smooth them to infer the $1 \sigma$ uncertainty. The planet is very unlikely to be Fe-only, in agreement with the previous conclusions (Léger et al 2009). In fact, CoRoT-7b is likely to have a smaller core in proportion to its size than do the terrestrial planets. The presence of ice is thought to be unlikely because of the proximity of the planet to its star, CoRoT-7, resulting in high surface temperatures. The nominal mass-radius measurement is consistent with a composition of rock only; given the uncertainties in the mass-radius measurement and relations, a metallic core cannot be ruled out.

Recent measurements of exoplanet GJ $1214 \mathrm{~b}$ give a radius of $2.65 \pm 0.11 \mathrm{R}_{\mathrm{E}}$ and mass of $6.55 \pm 0.9 \mathrm{M}_{\mathrm{E}}$ (Charbonneau et al 2009) or $6.45 \pm 0.9 \mathrm{M}_{\mathrm{E}}$ (Carter et al 2011), lying between the mass-radius curves for $\mathrm{H}_{2} \mathrm{O}$ and $\mathrm{CH}_{4}$. The closest analogue in the solar system is the icy giants; the mass and radius of GJ 1214b are consistent with a higher proportion of $\mathrm{H}_{2} \mathrm{O}$, or with a proportionately larger amount of rock or Fe, than Neptune or Uranus. The observations do not of themselves suggest an 'ocean planet' as the planet's density seems implausibly low. Furthermore, as has been pointed out previously (Adams et al 2008), a mean density consistent with a 'water world' could arise from a rocky interior with an $\mathrm{H} / \mathrm{He}$ atmosphere.

Recent parameters for the super-massive exoplanet HAT-P-2b are a mass of 
$9.09 \pm 0.24 \mathrm{M}_{\mathrm{J}}$ and a radius of $1.157_{-0.092}^{+0.073} \mathrm{R}_{\mathrm{J}}$ (Pál et al 2009). These parameters are consistent with a massive hydrogen-rich planet, as concluded previously. Proportionately, the radius is smaller with respect to our predicted mass-radius curve for hydrogen than are Jupiter or Saturn, suggesting a larger proportion of rock or Fe. A pure hydrogen planet of the nominal mass would have a radius of $1.12 \mathrm{MJ}$ and a central pressure of $214 \mathrm{TPa}$. The actual proportion of rock that would be consistent with the observed mass and radius is difficult to estimate using the available EOS, because they roll over at lower masses; future studies will include theoretical prediction and experimental investigation of the EOS under relevant conditions.

The super-massive exoplanet CoRoT-3b has a mass of $21.7 \pm 1 \mathrm{M}_{\mathrm{J}}$ and $1.01 \pm 0.07 \mathrm{R}_{\mathrm{J}}$ (Deleuil et al 2008), lying close to our mass-radius curve for $\mathrm{H}$.

Our mass-radius relation for $\mathrm{H}$ confirms the anomalously large radius of CoRoT-2b. As proposed recently (Leconte et al 2009), a plausible explanation is that this planet is young and hot, from accretion, tidal heating, or internal processes. The radius would be larger for a warmer isentrope, but is relatively insensitive to temperature. Another potential source of uncertainty, particularly for warm bodies with a significant proportion of volatiles, is the sensitivity to definition of the outer surface of the body. If the low-density gas in the outer region of the atmosphere has a higher opacity than expected, the planet would appear larger.

Using the location of a given planet with respect to the mass-radius relation for different compositions, it is possible to estimate the pressure in the center of the core from the corresponding mass-pressure relation (Fig. 12). Thus the central pressure of Kepler-10b is likely to be between 1.5 and $2.7 \mathrm{TPa}$, and that of CoRoT-7b is likely to be between 0.7 and $2 \mathrm{TPa}$, which are accessible using planar ramp-loading experiments at the OMEGA and NIF facilities. The core pressure in GJ 1214b is approximately 1 TPa: the same mass 
of $\mathrm{H}_{2} \mathrm{O}$ gives a pressure of around $1.5 \mathrm{TPa}$, and that mass of $\mathrm{CH}_{4}, 0.5 \mathrm{TPa}$. It would be most useful to improve EOS of ice compositions into this regime, by QMD simulations and dynamic loading experiments; again, planar ramp experiments at NIF should be able to access relevant states. The core pressure in HAT-P-7b is approximately $20 \mathrm{TPa}$, which may be accessible with subsequent NIF experiments using convergent compression. The core of CoRoT-3b is at the highest pressure known for non-stellar matter: $1900 \mathrm{TPa}$ according to the EOS used. Such pressures are achieved in inertial confinement fusion experiments, but will require very significant experimental developments before EOS experiments can be performed.

\section{Conclusions}

Mass-radius relations were derived using EOS widely used for, and thus validated against, dynamic compression experiments. The results were similar to relations used previously to infer planetary structures, but were not identical.

Mass-radius relations for Fe were inferred using EOS calculated to higher pressures than reported previously, using density functional theory. The difference between the cold curve and STP isentrope had a negligible effect on the mass-radius relation in the exoplanet regime. The effect of $\mathrm{Ni}$ in Fe was significant for the EOS, but negligible for the mass-radius relation of exoplanets. In a differentiated planet, the radius was more sensitive to small proportions of rock than has been concluded previously.

These results support the conclusion that CoRoT-7b, with measurements analyzed by

Léger et al (2009), is 'Earth-like' in that it is likely to have a rocky layer over an Fe-Ni core, though the present uncertainty in the exoplanet's mass is too large to constrain the mass of Fe significantly: there may be very little. GJ 1214b lies between the curves for $\mathrm{H}_{2} \mathrm{O}$ and 


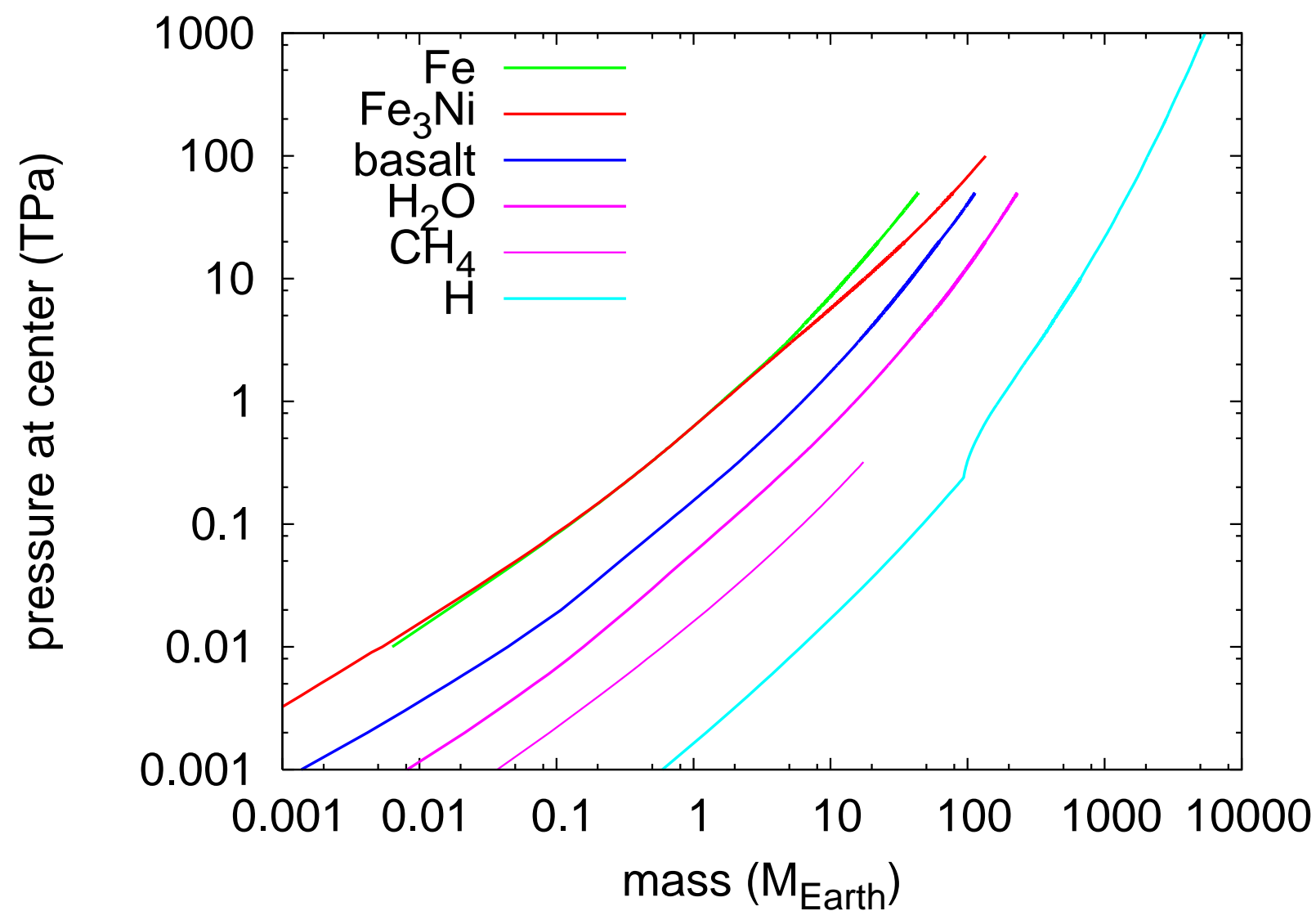

Fig. 12.- Mass-pressure relations for different classes of material. 
$\mathrm{CH}_{4}$, which are compositions where improvements to the EOS are particularly desirable. The composition cannot be constrained meaningfully from present data, and suggestions that it might be an 'ocean planet' are speculative. The present results also support the conclusion that HAT-P-2b is a gas giant, though the density is proportionately larger than the gas giants in the solar system, indicating a higher proportion of matter of higher atomic number. The mass-radius relations imply core pressures of $0.75 \pm 0.15 \mathrm{TPa}$ for CoRoT-7b, a range range $0.5-1.5 \mathrm{TPa}$ for GJ $1214 \mathrm{~b}$, over $210 \mathrm{TPa}$ in HAT-P-2b, and around $1900 \mathrm{TPa}$ in CoRoT-3b.

Based on the constituent physics and approximations, we conclude that current theoretical techniques for electronic structure, with care in the use of pseudopotentials, should be as suitable for predicting EOS in the exoplanet regime as they are at low pressures. Shock measurements are relevant in terms of the compressions that can be explored, though interpretation is involved to account for the elevated temperatures. Ramp loading techniques are very appropriate, and planned NIF experiments should provide data on Fe in the region where DFT mass-radius relations deviate from SESAME EOS. In both experiment and theory, the limiting cases of $\mathrm{Fe}$ (or Fe-Ni) and $\mathrm{H}$ are the subject of active research. Rocks and ices are more complicated in terms of composition and structure, and there is clearly a need for future research into the properties of these materials at compressions relevant for planets and exoplanets.

\section{Acknowledgments}

The authors would like to acknowledge the invaluable contributions of G.I. Kerley, J.D. Johnson at Los Alamos National Laboratory, W.M. Howard at Lawrence Livermore National Laboratory, and K. Rice at the Royal Observatory, Edinburgh. This work was performed under the auspices of the U.S. Department of Energy under contract 
DE-AC52-07NA27344. 


\section{REFERENCES}

Ackland, G.J. 2002, J. Phys.: Condens. Matter, 14, 2975

Adams, E.R., Seager, S., \& Elkins-Tanton, L. 2008, ApJ, 673, 1160

Ahrens, T.J., \& Gregson, J.V.G. 1964, J. Geophys. Res., 69, 4839-4874

Ahrens, J.J. 1966, J. Appl. Phys., 37, 2532-2541

Albers, R. \& Johnson, J.D. 1982, documentation for SESAME EOS 5251 for H, in Holian $(\underline{1984})$

Alfè, D., Gillan, M.J., \& Price, G.D. 2000, Nature, 405, 172

Alfè, D., Kresse, G. \& Gillan, M.J. 2000, Phys. Rev. B, 61, 132

Alfè, D., Price, G.D., \& Gillan, M.J. 2001, Phys. Rev. B, 64, 045123

Barnes, J. \& Rood, J. 1973, (Los Alamos National Laboratory), documentation for SESAME EOS 2140 for Fe, in Holian (1984)

Barnes, J.F. et al 1974, J. Appl. Phys., 45, 727

Barnes, J.F. \& Lyon, S.P. 1988, (Los Alamos National Laboratory), documentation for SESAME EOS 7460 for MgO, in Lyon \& Johnson (1992)

Barnes, J.F. \& Lyon, S.P. 1988, SESAME Equation of State Number 7530, Basalt, Los Alamos National Laboratory report LA-11253-MS

Bastea, S., Fried, L.E., Howard, W.M., Souers, P.C., \& P.A. Vitello, P.A. 2007, CHEETAH computer program (current version is 5.0, Lawrence Livermore National Laboratory release number UCRL-CODE-234077) 
Belonoshko, A.B., Dorogokupets, P.I., Johansson, B., Saxena, S.K., \& Koi, L. 2008, Phys. Rev. B, 78, 104107

Batalha, N.M. et al 2011, ApJ, 729, 27

Bradley, D.K. et al 2009, Phys. Rev. Lett., 102, 075503

Bushman, A.V., Kanel', G.I., Ni, A.L., \& Fortov, V.E. 1993 Intense Dynamic Loading of Condensed Matter (Taylor \& Francis, London)

Caracas, R. \& Cohen, R.E. 2008, PEPI, 168, 3-4, 147 and references therein

Carter, J.A., Winn, J.N., Holman, M.J., Fabricky, D., Berta, Z.K., Burke, C.J., and Nutzman, P. 2011, ApJ, 730, 82

Charbonneau, D. Brown, T.M., Latham, D.W., \& Mayor, M. 2000, ApJ, 529, L45-48

Charbonneau, D., Brown, T.M., Noyes, R.W., \& Gilliland, R.L. 2002, ApJ, 568, 1, 377

Charbonneau, D. et al 2009, Nature, 462, 891

Chau, R., Hamel, S., \& Nellis, W.J. 2011, Nature Comm., 2, 203

Clerouin, J. 2002, J. Phys.: Condens. Matter, 14, 9089

Delaney, K., Pierleoni, C. \& Ceperley, D.M. 2006, Phys. Rev. Lett., 97, 235702-1-4

Deleuil, M. et al 2008, A\&A, 491, 889

Drummond, N.D., \& Ackland, G.J. 2002, Phys. Rev. B, 65, 184104

Edwards, J. et al 2004, Phys. Rev. Lett., 92, 075002

Ferraz-Mello, S., et al 2011, A\&A, 531, A161

Fortney, J.J., Marley, M.S., \& Barnes, J.W. 2007, ApJ, 668, 1267 
Grasset, D., Schneider, J., \& Sotin, C. 2009, ApJ, 693, 722

Hamel, S., Morales, M., \& Schwegler, E. 2011, Phys. Rev. B (in press)

Henry, G.W. et al. 2000, ApJL, 529, 1, L41

Hohenberg, P. \& Kohn, W. 1964, Phys. Rev. B, 136, 3B, 864

Holian, K.S. (Ed.) 1984, Los Alamos National Laboratory report LA-10160-MS Vol 1c

Holst, B., French, M, and Redmer, R. 2011, Phys. Rev. B, 83, 235120

Haar, L. \& Gallagher, J.S. 1978, J. Phys. Chem. Ref. Data, 7, 3, 635

Hatzes, A. et al 2010, A\&A, 520, A93

Hensel, F. 1999, J. Non-Crystalline Solids, 150-152, 135-138

Hicks, D.G., Boehly, T.R., Celliers, P.M., Eggert, J.H., Vianello, E., Meyerhofer, D.D., \& Collins, G.W. 2005, Phys. Plasmas, 12, 082702

Johnson, J.D. 1984, documentation for SESAME EOS 5502 for $\mathrm{CH}_{4}$, in Holian (1984)

Johnson, J.D. \& Lyon, S.P. 1984, documentation for SESAME EOS 7383 for $\mathrm{SiO}_{2}$, in Lyon \& Johnson (1992)

Johnson, J.D. \& Lyon, S.P. 1990, documentation for SESAME EOS 7154 for $\mathrm{H}_{2} \mathrm{O}$, in Lyon \& Johnson (1992)

Kerley, G.I. 1972, Los Alamos National Laboratory report LA-4776

Kerley, G.I. 1980, J. Appl. Phys., 51, 10, 5369

Kerley, G.I. 1993, Sandia National Laboratories report SAND93-0227

Kittel, C. \& Kroemer, H. 1980, Thermal Physics (W.H. Freeman, New York). 
Knudson, M.D., \& Desjarlais, M.P. 2009, Phys. Rev. Lett., 103, 225501

Kohn, W. \& Sham, L.J. 1965, Phys. Rev., 140, 4A

Leconte, J., Baraffe, I., Chabrier, G., Barman, T., \& Levrard, B. 2009, A\&A, 506, 385

Léger, A. et al 2009, A\&A manuscript 11933, preprint arXiv:0908.0241v3

Liberman, D.A. 1979, Phys. Rev. B, 20, 12, 4981

Lindl, J.D. 1998, Inertial Confinement Fusion (Springer-Verlag, New York)

Loeillet, B. et al 2008, A\&A, 481, 529

Lorenzen, W, Holst, B, \& Redmer, R. 2010, Phys. Rev. B, 82, 195107

Luo, S.-N., Swift, D.C., Mulford, R.N., Drummond, N.D., \& Ackland, G.J. 2004, J. Phys.: Cond. Matt., 16, 30, 5435

Lyon, S.P. \& Johnson, J.D. 1992, Los Alamos National Laboratory report LA-UR-92-3407

Mayor, M. \& Queloz, D. 1995, Nature, 378, 355-359

McQueen, R.G., Marsh, S.P., Taylor, T.W., Fritz, J.N., \& Carter, W.J. 1970, in High Velocity Impact Phenomena, ed. R. Kinslow, (Academic Press, New York)

Michielsen, K. \& De Raedt, H. 1996, Europhys. Lett., 34, 6, 435

Militzer, B. 2009, Phys. Rev. B, 79, 155105-1-18

Morales, M.A., Schwegler, E., Ceperley, D.M., Pierleoni, C., Hamel, S., \& Caspersen, K. 2009, PNAS, 106, 1324 and references therein

Morales, M.A., Pierleoni, C., Schwegler, E., \& Ceperley, D.M. 2010, hydrogen from ab initio simulations PNAS, 107, 12799-12803 
Mulford, R.N. \& Swift, D.C. 2006, Proc. Am. Phys. Soc. Topical Conf. on Shock Compression of Cond. Matt., Conf. Proc. 845 pp.461-464 (Am. Inst. Phys.)

Pál, A. et al 2009, MNRAS, 401, 4, 2665-2674

Payne, M.C., Teter, M.P., Allan, D.C., Arias, T.A. \& Joannopoulos, J.D. 1992, Rev. Mod. Phys., 64, 4

Perdew, J. 1992, Phys. Rev., B46, 6671

Perdew, J. 1994, Phys. Rev., B50, 4954

Pont, F., Aigrain, S., \& Zucker, S. 2011, MNRAS, 411, 1953

Press, W.H., Flannery, B.P., Teukolsky, S.A. \& Vetterling, W.T. 1989, Numerical Recipes (Cambridge University Press, London)

Ragan III, C.E. 1984 Phys. Rev. A, 25, 6, 3360

Recoules, V., Lambert, F., Decoster, A., Canaud, B., \& Clerouin, J. 2009, Phys. Rev. Lett., $102,075002-1-4$

Reisman, D.B. et al 2001, J. Appl. Phys., 89, 1625

Salpeter, E.E., \& Zapolsky, H.S. 1967, Phys. Rev., 158, 876

Saumon, D. \& Chabrier, G. 1992 Phys. Rev. A, 46, 2084

Schneider, J. 2011, The Extrasolar Planets Encyclopaedia (version 2.06), http://exoplanet.eu and references therein

Seager, S., Kuchner, M., Hier-Majumder, C.A., \& Militzer, B. 2007 ApJ, 669, 1279

Sola, E., Brodholt, J.P., \& Alfè, D. 2009, Phys. Rev. B, 79, 024107 
Stacey, F.D., \& Davis, P.M. 2004, PEPI, 142, 137-184

Stixrude, L. \& Cohen, R.E. 1995, Science, 267, 1972

Stixrude, L., de Koker, N., Mookherjee, M., \& Karki, B.B. 2009, EPSL, 278, 226-232

Stixrude, L. \& Lithgow-Bertelloni, C. 2010, Rev. Mineralogy \& Geochem., 71, 465

Stixrude, L. \& Lithgow-Bertelloni, C. 2011, Geophys. J. Int., 184, 1180

Swift, D.C., Ackland, G.J., Hauer, A., \& Kyrala, G.A. 2001, Phys. Rev. B, 64, 214107

Swift, D.C. \& Johnson, R.P. 2005, Phys. Rev. E, 71, 066401

Swift, D.C. et al 2005, J. Appl. Phys., 98, 093512

Swift, D.C., Paisley, D.L., McClellan, K.J., \& Ackland, G.J. 2007, Phys. Rev. B, 76, 134111

Swift, D.C. 2008, J. Appl. Phys., 104, 7, 073536

Swift, D.C. 2009, Sensitivity of rocky planet structures to the equation of state, preprint arXiv:0908.3294

Swift, D.C. 2010, Equations of state for Fe and Fe-Ni to massive exoplanet core conditions, in preparation

Swift, D.C. 2011, Grüneisen equation of state for ammonia, in preparation.

Troullier, N., \& Martins, J.L., 1991, Phys. Rev. B, 43, 1993

Valencia, D., Ikoma, M., Guillot, T., \& Nettelmann, N. 2010, A\&A, 516, A20

van Thiel, M. 1966, Compendium of Shock Wave Data, Lawrence Radiation Laboratory report UCRL-50108

Wasserman, E., Stixrude, L., \& Cohen, R.E. 1996, Phys. Rev. B, 53, 8296 
Wentzcovitch, R.M., Wu, Z., \& Carrier, P. 2010, Rev. Mineralogy \& Geochem., 71, 99

Wolszczan, A. \& D.A. Frail 1992, Nature, 355, 145 